\title{
Immunohistochemistry and Tissue Microarray Techniques to Demonstrate Human Papilloma Virus Antigen and Cell Cycle Proteins in Squamous Intraepithelial Lesions and Cancer
} Cervix

\author{
Rama Bajoriya ${ }^{1}$, Manjiri Makde ${ }^{2}, \mathrm{MM} \mathrm{Kamal}^{3}$
}

\section{Abstract}

Cervical cancer remains the second most common cancer among women of developing countries. Human papilloma virus (HPV) infection has been identified as leading etiological agent for cervical cancer. Cell cycle dysregulation has a central role in cervical cancer carcinogenesis. p16 ${ }^{\text {INK4 }}$ is a tumor-suppressor protein whose increased expression has been associated with HPV-infected dysplastic epithelium and cancer of cervix. $\mathrm{Ki}-67$ is a non-histone protein expressed in nucleus during whole cell cycle except $\mathrm{G}_{0}$ and $\mathrm{G}_{1}$. It constitutes an efficient marker of proliferating cells. It has its utility in distinguishing non-diagnostic atypia from squamous intraepithelial lesion (SIL). Our aim was to evaluate the usefulness of $16^{\mathrm{INK} 4}$ and Ki-67 in the diagnosis of spectrum of SIL cervical carcinoma, to know the genotype of HPV positivity in invasive cervical cancers using the TMA technique and to demonstrate the importance of the technique of tissue microarray (TMA) as an educational activity. Paraffin blocks, histology slides, and clinical data of 354 cervical biopsies sent to department's histology laboratory at GMC Nagpur were retrieved. The lesions were classified as LSIL, HSIL, and cervical carcinoma as per WHO guidelines. All cases of adenocarcinoma and randomly selected cases of squamous cell carcinoma were subjected to $\mathrm{p} 16^{\text {INK4 }}$ and Ki-67 labeling using TMA technique. Ki- 67 and p16 ${ }^{\text {INK4 }}$ are aberrantly expressed in LSIL-HSIL-carcinoma sequence. p16 has significant association with severity of histological lesions. Also, HPV positivity with 16 and 18 was $100 \%$ in all the carcinomas.

Keywords: Cancer cervix, Human papilloma virus 16 and 18, Ki67, p16 INK4a , Squamous intraepithelial lesion, Tissue microarray. Journal of South Asian Federation of Obstetrics and Gynaecology (2020): 10.5005/jp-journals-10006-1780
\end{abstract}

\section{Respected Sir,}

Carcinoma cervix is the second most common cause of mortality and morbidity in women of developing countries. Human papilloma virus (HPV) is identified as an essential cause of cervical cancer. Cell cycle dysregulation plays a central role in cervical carcinogenesis. The tumor-suppressor protein $\mathrm{p} 16^{\mathrm{INK} 4 \mathrm{a}}$ is overexpressed and is linked to the oncogenic transformation caused by persistent highrisk HPV infection. It points to the inactivation of the cell cycle control. On the contrary, Ki67 is a proliferation marker confined to the parabasal cell layer of stratified squamous epithelium. It is a non-histone protein expressed in nucleus during whole cell cycle except $\mathrm{G}_{0}$ and $\mathrm{G}_{1}$.

It is an established fact that the tissue microarray (TMA) technique allows simultaneous evaluation of multiple tissue samples for gene expression analysis. The recipient block can accommodate samples from hundreds of different donor specimens and these can be stained simultaneously. ${ }^{2}$ This advantage has been brought to use to evaluate the expression of p16 ${ }^{\text {INK4a }}$ and Ki67 biomarkers using the cervical biopsies.

We conducted a study of 18 months' duration at Government Medical College and Hospital, Nagpur, with the primary aim to demonstrate the nature of positivity of p16 ${ }^{\text {INK4a }}$ and Ki67 in the spectrum of cervical lesions from metaplasia to low-grade squamous intraepithelial lesion(LSIL) [HPV and cervical intraepithelial neoplasia (CIN) I] and high-grade squamous intraepithelial lesion (HSIL) (CIN II and III) and to know the genotype of HPV positivity in invasive cervical cancers using the TMA technique. The secondary aim was to educate the staff and students regarding the utility and interpretation of TMA sections.

\footnotetext{
1,2Department of Pathology, Government Medical College, Nagpur, Maharashtra, India

${ }^{3}$ Department of Pathology, Public Health Department, Yavatmal, Maharashtra, India
}

Corresponding Author: Manjiri Makde, Department of Pathology, Government Medical College, Nagpur, Maharashtra, India, Phone: +91 9665444920, e-mail: majiri0288@gmail.com

How to cite this article: Bajoriya $R$, Makde $M$, Kamal MM. Immunohistochemistry and Tissue Microarray Techniques to Demonstrate Human Papilloma Virus Antigen and Cell Cycle Proteins in Squamous Intraepithelial Lesions and Cancer Cervix. J South Asian Feder Obst Gynae 2020;12(2):116-117.

Source of support: Nil

Conflict of interest: None

The study material included the clinical data and histology slides from 354 cervical biopsies sent to our department. Two hundred and thirty-seven biopsies were from clinically invasive cancers and 117 were colposcopically directed biopsies. Slides from these biopsies were reviewed and the paraffin blocks were retrieved for the TMA technique. The distribution of cases for colposcopically directed biopsies was as follows-benign 94, LSIL 8, and HSIL 15. Of the invasive cancers, 7 were adenocarcinomas, 2 were malignant mixed Mullerian tumors (MMT), 1 was adenosquamous carcinoma, and the rest 227 were squamous cell carcinomas. Of the 117 cervical biopsies from clinically noninvasive lesions, we selected 3 cases of LSIL and 4 cases of HSIL and subjected them to Ki67 and p16. ${ }^{\text {INK4a }}$ 
The selection criterion of these biopsies was that they had strips of squamous metaplastic epithelium in addition to the histological features of LSIL and HSIL. Forty cases comprising of 07 adenocarcinoma, 02 MMT, 1 adenosquamous carcinoma, and 30 selected cases of squamous cell carcinoma were also subjected to immunohistochemistry (IHC) for p16 $6^{\mathrm{INK} 4 \mathrm{a}}$ and Ki67 and polyclonal antigen detection of HPV 16 and 18 using TMA technique.

The $16^{\text {INK4a }}$ expression in squamous epithelium is variable, and may express as weak to strong positivity, in both the nucleus and the cytoplasm. We observed that normal stromal and squamous epithelium were negative for p16 ${ }^{\text {INK4a }}$, whereas clear and distinct staining was positive in dysplastic cells and malignant cells. The intensity and proportion of staining increased with increasing grade of CIN I to CIN III. One out of three cases of LSIL was positive for $\mathrm{p} 16^{\mathrm{INK} 4 \mathrm{a}}$, whereas the remaining two were negative for the same. All the four cases of HSIL were positive for p16 ${ }^{\text {INK4a }}$. The associated metaplastic epithelium in all these seven biopsies was weakly positive for p16 ${ }^{\text {INK4a }}$. p16 $6^{\text {INK4a }}$, the surrogate marker for high-risk HPV infection, was positive in all the invasive carcinomas. Also, HPV positivity with 16 and 18 was $100 \%$ in all the carcinomas.

The expression or immunoreactivity for Ki67 cells is in the form of dark brown staining exclusively limited to the nucleus. Normally, only parabasal cell nuclei are positive for Ki67 which can be taken as an in-built control. On applying this marker, we found that Ki67 positive nuclei were scattered in upper epithelial layer for LSIL lesions, whereas diffuse involvement of middle and upper layers of squamous epithelium was seen in HSIL. In the metaplastic epithelium, the positivity was $<15 \%$. With increasing grade of $\mathrm{CIN}$, the number of Ki67 positive cells also increased. Ki67 was positive in all the cases of invasive carcinomas. However, Ki67 alone does not discriminate HPV-mediated dysplasia vs proliferating cells in benign reactive process and immature squamous metaplasia.
Even though Ki67 positive nuclei are seen scattered in all the layers of the reactive and metaplastic epithelium, the $\mathrm{p} 16^{\mathrm{INK} 4 \mathrm{a}}$ remains negative. ${ }^{3}$ In view of this, we recommend using both Ki67 and p16 ${ }^{\text {INK4a }}$ as complementary markers for differentiation between dysplastic and nondysplastic lesions. Both in combination are also suited to triage borderline cervical abnormalities as compared to high-risk HPV DNA testing.

In this study, TMA demonstrated uniform positivity for HPV 16 and 18 irrespective of the histological type of cervical cancer. Also, the p16 ${ }^{\text {INK4a }}$ was predominantly expressed in oncogenic process. Ki67 proliferation marker and its positivity when seen in upper layers makes it a good predictor of squamous intraepithelial lesion (SIL). However, Ki67 alone cannot be used as single specific marker of underlying SILs. ${ }^{4}$ Gray zone lesions like ASC-H (atypical squamous cells-cannot rule out high-grade lesion), atypical squamous metaplasia, or atrophy are generally confused with HSIL. In such situations, utilizing both these markers in conjunction with morphological impression helps to achieve the final diagnosis.

\section{References}

1. Conesa-Zamora P, Doménech-Peris A, Orantes-Casado FJ, et al. Effect of human papillomavirus on cell cycle related proteins p16, ki67, cyclin D1, p53 and ProEx C in precursor lesions of cervical carcinoma: a tissue microarray study. Am J Clin Pathol 2009;132(3):378-390. DOI: 10.1309/AJCPOOWY1VIFCYDC.

2. Lesnikova I, Lidang M, Hamilton-Dutoit S, et al. p16 as a diagnostic marker of cervical neoplasia: a tissue microarray study of 796 archival specimens. Diagn Pathol 2009;4:22. DOI: 10.1186/1746-1596-4-22.

3. Walts AE, Bose S.P16/Ki67 immunisations is useful in stratification of atypical metaplastic epithelium of cervix. Clin Med Pathol 2008;1:3642. DOI: $10.4137 / C P a t h . S 522$.

4. Malpica A, Deavers M, Euscher E. Biopsy interpretation of the uterine cervix and corpus. Squamous lesions of uterine cervix. 2010;2:17-27. 